\title{
Nodal prices in an integrated energy system
}

\section{Ana M. Quelhas*, Esteban Gil and James D. McCalley}

Department of Electrical and Computer Engineering, Iowa State University, Ames, 50011, IA USA

E-mail: aquelhas@iastate.edu_E-mail: tebagil@iastate.edu

E-mail: jdm@iastate.edu

${ }^{*}$ Corresponding author

\begin{abstract}
This paper presents a multiperiod generalised network flow model used to analyse the economic interdependencies of integrated energy systems comprising the electric network and the various fuel supply and delivery systems in a medium term operational time frame. By using a network flow programming model, one can take advantage of much faster solution procedures than standard linear programming techniques; an issue of importance considering the dimensionality of such integrated systems. The nodal prices that are obtained as a byproduct of the optimisation algorithm provide a way to analyse the economic interdependencies between the various fuel networks and the electric network. A numerical example is presented to highlight the benefits of the methodology and illustrate how nodal prices in the electric network are influenced by the dynamics of the various fuel networks.
\end{abstract}

Keywords: critical infrastructures; generalised network flow model; integrated energy networks; nodal pricing; optimisation.

Reference to this paper should be made as follows: Quelhas, A.M., Gil, E. and McCalley, J.D. (2006) 'Nodal prices in an integrated energy system', Int. J. Critical Infrastructures, Vol. 2, No. 1, pp.50-69.

Biographical notes: Ana M. Quelhas is a $\mathrm{PhD}$ candidate in Electrical and Computer Engineering at Iowa State University. She graduated from the Faculdade de Engenharia, Universidade do Porto, Portugal, in Electrical and Computer Engineering (1999). She received her MS degree in Electrical Engineering from Iowa State University in 2001, and another MS degree in Economics, also from Iowa State University, in 2004.

Esteban Gil is a PhD candidate in Electrical and Computer Engineering and a MS student in Statistics at Iowa State University. He received his BS and MS degrees in Electrical Engineering from Universidad Técnica Federico Santa María (UTFSM), Valparaíso, Chile, in 1997 and 2001, respectively.

Dr. James D. McCalley received his BS, MS, and $\mathrm{PhD}$ degrees from the Georgia Institute of Technology, Atlanta, in 1982, 1986 and 1992, respectively. He was employed with the Pacific Gas and Electric Company, San Francisco, CA, from 1985 to 1990 . He is a Professor in the Electrical and Computer Engineering Department at Iowa State University, where he has been since 1992. He is a fellow of the IEEE and a registered Professional Engineer in California. 


\section{Introduction}

The economic efficiency of the electric energy system depends not only on the performance of the electric generation and transmission subsystems, but also on the ability to produce and transport the various forms of primary energy, particularly gas, coal and petroleum. However, most of the medium term electric energy models, designed to address various issues associated with the operation of electric power systems, exclude the primary energy flows from their formulation, or simply represent them as exogenous variables that follow an assumed behaviour dictated by fuel contracts. This has been partly due to the difficulty of formulating models capable of analysing the integrated system while accounting for characteristics unique to each subsystem.

Today's industrial climate in the USA motivates a more integrated study of the energy system.

- As the electric power industry becomes more competitive, economic performance of electricity delivery is intensely scrutinised from a national perspective, with electricity delivery price as a key metric (Energy Information Administration, 2000). Customers and regulators are questioning electricity markets in which prices are significantly higher than those in other parts of the country, resulting in heavy pressure to identify means to lower prices.

- Long term contracts have become shorter in duration, as electric power generators are trying to pass market risks on to primary energy suppliers (producers and carriers) (Energy Information Administration, 1998). This increases concern on the part of generation owners that they may be more vulnerable to short or medium term contingencies in fuel supply.

- The perception has grown that the economic integrity of the energy system depends heavily on the interdependencies between its constituent subsystems, and meaningful economic analysis must account for these interdependencies (Knight et al., 1998; Rinaldi et al., 2001).

In this paper, we propose a multiperiod generalised network flow model to represent an integrated electric energy system having fuel mix similar to that of the USA, where the production, transportation and delivery of fossil fuels to the power plants are modelled for a medium term analysis (several months to two years) of the overall economic performance of the energy flows. In $2003,50 \%$ of the net electricity generated in the USA came from coal (Energy Information Administration, 2004). Natural gas and petroleum accounted for $17 \%$ and $3 \%$, respectively. Combined, the fossil fuels were responsible for approximately $70 \%$ of the nation's electric energy generated. The three primary energy forms fully incorporated in the model (coal, gas, and petroleum), together with the electric energy subsystem, have the common characteristic that they are moved via a network transportation system from their sources of production to where they are used. The schedules of electricity generated from nuclear energy and renewable energy forms are given exogenously and represented as direct inputs into the electric transmission system (Sterman, 2000). Despite the relative importance of electricity generation from nuclear energy (roughly $20 \%$ of the electricity net generation in the USA in 2003), this energy form is associated with large time constants (slow dynamics), and therefore is assumed not to influence the medium term analysis proposed in this paper. 
As regards renewable energies, they comprise a relatively small percentage of electric fuel supply. In addition, they cannot be transported as a raw fuel and therefore represent no energy movement alternative to electric transmission in the way that coal, gas and petroleum do.

There has been significant work in scheduling fuel deliveries in order to optimise electric energy production, and a representative sample includes (Wood and Wollenberg, 1996; Vermuru and Lemonidis, 1990; Moslehi et al., 1991). The common denominator of the known fuel scheduling approaches is that they view the fuel system only in terms of contracts and associated penalties for possible violations. In other words, there has been little effort in optimising the electric power system operations accounting for fuel production, storage and transportation costs and capabilities. Our approach involves the development and study of an integrated, interdependent energy system model that includes the coal, gas, petroleum, and electric transportation networks, rather than the optimisation of the performance of a single energy subsystem.

In the context of the electric system, the concept of nodal prices (also referred to as locational marginal prices, or LMP, in power industry terminology) has become more and more familiar, as several electricity markets have adopted some version of this approach to price the electric energy and to improve the efficient usage of the power grid and congestion management (Sun, 2002; Tabors, 2001; Hong and Weng, 1999; Ott, 2003; Litvinov et al., 2004; Motto et al., 2002). In contrast with a single price mechanism, under a nodal pricing scheme, prices vary from node to node because of transmission line congestion and losses (Chen et al., 2002), and they can therefore be used to determine the value of transmission rights and to provide economic signals for generation and transmission investments (Shahidehpour et al., 2002).

The purpose of this paper is to describe a model developed to enable analysis of how nodal prices in the electric network are influenced by the dynamics of the various fuel networks. Knowledge and understanding of these interdependencies is expected to induce the most economically efficient use of fuel production, fuel storage, fuel transportation, electricity generation and demand and transmission resources, through the correct economic signals provided. The concept of nodal prices is expanded to an integrated energy system by optimising the energy flows in a generalised network flow model that explicitly represents the electric subsystem together with the various fossil fuel networks in a single mathematical framework. Since all entities involved in the operation of the electric energy system are fully represented, the nodal prices obtained as a byproduct of the optimisation procedure provide a means to identify the interdependencies between the fuel subsystems and the electric subsystem.

The remaining sections are organised as follows. Section 2 presents a generalised network flow formulation of the integrated energy system. Section 3 describes the computation of nodal prices. Section 4 provides numerical results of a case study to highlight the benefits of the methodology proposed. Section 5 gives concluding remarks. 


\section{Modelling approach}

\subsection{Network flow model}

Conceptually, the integrated energy model is a simplified representation of the coal, gas, petroleum and electricity systems, structured as a network composed of nodes and arcs, with energy flowing from node to node along paths in the network. Such a structure lends itself nicely to the adoption of network flow programming (Glover et al., 1992). When a situation can be entirely modelled as a network, very efficient algorithms exist for the solution of associated optimisation problems; many times more efficient than linear programming in the utilisation of computer time and memory resources (Jensen and Bard, 2002). Our network flow model of the integrated energy system falls into the category of generalised minimum cost flow problems (Ecker and Kupferschmid, 1991) and can be solved by applying the generalised network simplex algorithm. Although the worst case complexity for the generalised network simplex algorithm is exponential, in practice, the running time of this algorithm is a lower order polynomial in $n$ and $m$, where $n$ is the number of nodes and $m$ is the number of arcs. A careful implementation of the generalised network simplex algorithm provides the optimal solution in less than $O(\mathrm{~nm})$ time. Empirical investigations have further determined that the generalised simplex algorithm is only two to three times slower than the network simplex algorithm for the pure minimum cost flow problem (Ahuja et al., 1993).

The scenario of this generalised minimum cost flow problem is the following. The supply node (source node) has an excess of coal, gas, or petroleum, while the demand nodes (sink nodes) require certain amounts of electric energy. The remaining nodes (transhipment nodes) neither require nor supply the commodity (energy), but serve as a point through which energy passes; storage nodes are of this type. The energy flows through arcs that connect the nodes and there is conservation of energy at the nodes, implying that the total flow entering a node must equal the total flow leaving the node. The arc flows are the decision variables of the network flow programming model, measured in energy terms, e.g., MW week equivalent. Associated with each arc $(i, j)$ are the following parameters:

- lower bound, $e_{i j . m i n}$, (which can be zero) on the flow

- upper bound, $e_{i j . m a x}$, on the flow (also called capacity)

- $\quad c o s t, c_{i j}$, per unit of flow (which is the criterion for optimality)

- efficiency, $\eta_{i j}$, (sometimes called gain or loss factor) which multiplies the flow at the beginning of the arc to obtain the flow at the end of the arc.

The interpretation of the efficiency parameter is the following: when 1 unit of flow is sent on $\operatorname{arc}(i, j), \eta_{i j}$ units of flow arrive at node $j$ (Bazaraa et al., 1990). It is a positive rational number that represents losses if $\eta_{i j}<1$ or gains if $\eta_{i j}>1$. A network in which all arcs have unit gains is called a pure network. If some gains have values other than 1, the network is a generalised network.

The goal of the network flow problem is to satisfy electric energy demands with available fossil fuel supplies at the minimal total cost without violating the bound constraints. The costs considered are the fossil fuel production, transportation, and storage costs and the energy conversion (electricity generation) costs. 


\subsection{Node and arc definitions}

The network flow model of the integrated energy system comprises the following nodes:

- Source node. The source node is an artificial node that supplies all the energy necessary to satisfy the electric energy demand. Supply cannot be specified a priori, because it depends on the losses of the entire system, which in turn depend upon the flows.

- Transhipment nodes. The transhipment nodes represent the primary energy production facilities (coal mines, gas and oil wells), the storage facilities (coal piles, gas reservoirs and oil tanks), and the energy conversion facilities (power plants).

- Sink nodes. The sink nodes represent the control areas as defined by the North American Electric Reliability Council (http://www.nerc.com/ filez/rs.html), and each one is associated with a given demand.

Nodes may also represent an aggregation of different facilities with identical characteristics. For example, a coal supply node may represent an aggregation of several coal mines located in a particular region or zone, according to the level of granularity desired. Nonetheless, under the terminology that follows from the discipline of network flows and adopted in this paper, the price obtained as a byproduct of the optimisation procedure for this particular node is called the nodal price, although it can also be interpreted as a regional or zonal price.

The outgoing arcs of the dummy source node represent the production of coal, gas, and petroleum and imports of coal, gas, petroleum and electricity. The arcs represent the major transportation routes and associated transportation modes between the different facilities. In the coal and petroleum subsystems, the transportation modes include barges, railroads, trucks, pipelines and conveyers. In the gas subsystem, the arcs represent gas pipelines. Arcs also represent storage injections and withdrawals and inventories carried over between two consecutive time periods. In the electric subsystem, the arcs represent the connections between the different generators and their respective control area and the transmission lines among the control areas (tie lines). Energy losses in the production, storage and transportation of the primary energy forms, losses in the energy conversion process at the power plants and losses in tie lines are represented by appropriately chosen efficiency parameters on the arcs. Fuel production costs (extraction and processing charges) are associated with the outgoing arcs from the dummy source node; coal transportation rates and pipeline tariffs are assigned to the respective transportation arcs; storage fees are allocated to the arcs representing storage withdrawals; operation and maintenance costs of power plants are assigned to the arcs connecting the power plant nodes to the control area nodes; wheeling charges, or transmission costs associated with electric trade are allocated to the arcs representing tie lines.

Since the electricity demand is modelled at the level of the of control areas, the only transmission lines represented in the model are the tie lines, whose flows can be considered as decision variables, since the control areas have the capability of controlling the imported/exported energy flow with their adjacent control areas. In contrast, the energy flows in the transmission lines within a control area can not be considered as decision variables, because they are determined according to the Kirchhoff's laws. As a result, only bulk power (wholesale) transactions are considered. 


\subsection{Tie line representation}

A tie line is an undirected edge, because the energy can flow in both directions. Since the network flow model requires directed arcs, the transformation in Figure 1 shows an equivalent model where each undirected edge is replaced by an oppositely directed pair of arcs. If the flow in either direction has a lower bound of value 0 and the arc cost is nonnegative, in some optimal solution one of the flows in the directed arcs will be zero, which guarantees a nonoverlapping solution.

Figure 1 Representation of tie lines: (a) undirected edge and (b) oppositely directed pair of arcs

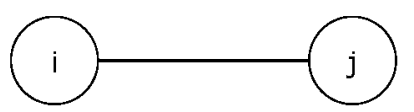

(a)

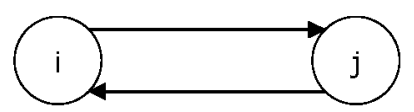

(b)

\subsection{Restrictions on nodes}

In a standard network flow model, the only parameters associated with the nodes are the supply or demand specified at the source or sink nodes, respectively. In the integrated energy system, resources such as power plants and storage facilities have restrictions on the flow that can pass through them (e.g., capacities, efficiency rates, and costs), which are parameters associated with arcs in a network flow model.

The transformation into a standard network flow model is done by replacing each of these nodes by a pair of nodes with an arc connecting them. The parameters of this arc dictate the restrictions on the flow that passes through the respective facility. Figure 2 illustrates this transformation, where the parameters $e_{\min }, e_{\max }, c$, and $\eta$ refer to the lower bound, upper bound, cost and efficiency, respectively, of the facility originally represented by node $i$.

Figure 2 Representation of restrictions on nodes: (a) node with restrictions and (b) equivalent network flow model

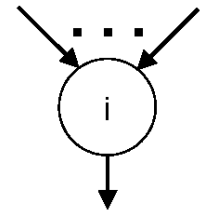

(a)

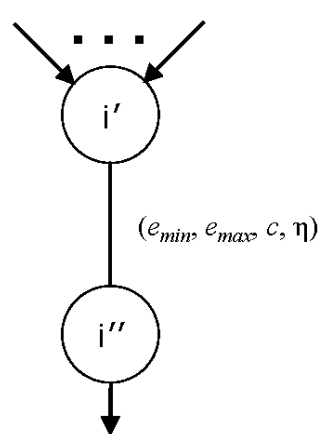

(b)

\subsection{Linearisation of costs and efficiencies}

A typical input-output characteristic of a steam turbine generator can be represented by a convex curve (Wood and Wollenberg, 1996). When multiplied by the fuel cost, we obtain the generating unit cost as a convex function of the flow. Total cost functions can then be 
approximated by piecewise linear functions that lead to step incremental cost functions. In a network flow representation, each linearisation segment is modelled by an arc, with the number of arcs determining the accuracy of the approximation. To illustrate this idea, we consider an arc that carries flow between nodes $i$ and node $j$. The cost associated with the flow in this arc is a convex function and can be fitted by a piecewise linear cost function, as shown in Figure 3(a). This cost function tells us that the first 20 units of flow have a unit cost of $\$ 2.5$, the next 10 units of flow have a unit cost of $\$ 5$, and any additional amount has a unit cost of $\$ 10$, up to the capacity of 40 units of flow. In a network flow model, this situation is represented using a set of arcs, one for each segment of the piecewise linear cost function, as illustrated in Figure 3(b). Because the unit costs are increasing, we have the guarantee that the flow in a given arc will only be positive if all the other arcs with smaller unit costs have reached their capacity limits, which assures the feasibility of the solution.

Figure 3 Representation of convex cost functions: (a) convex cost function and (b) equivalent network flow model

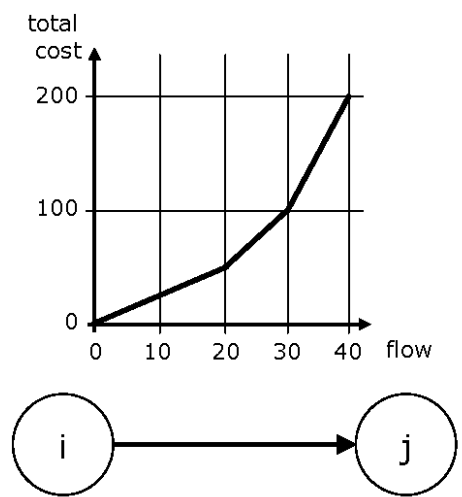

(a)

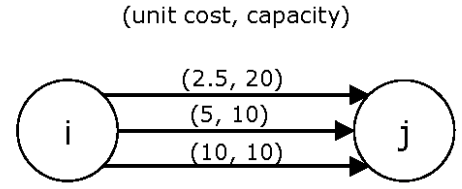

(b)

Nonconvex cost functions, in particular those associated with the input-output characteristics of combined cycle gas turbines, cannot be addressed with network flow programming techniques, and are therefore approximated by linear or piecewise linear convex functions. Although optimisation techniques capable of dealing with nonconvexities are available (Arroyo and Conejo, 2000), the cost in modelling complexity outweighs the improvement in model fidelity, considering that the level of aggregation intended (to the control area level) and convex approximations to nonconvex cost curves are reasonable.

Efficiency parameters may also be modelled using piecewise linear functions of the flow and can be represented by the multiple arc transformation illustrated above for convex cost functions. For example, power losses along the transmission lines are proportional to the square of the flow and efficiency can therefore be approximated by a piecewise linear function where the slopes decrease with the flow. In this situation, we have the guarantee that the arcs with the higher efficiency parameters (lower losses) will be filled up first, since they require the smallest amount of flow, and thus the smallest cost, for the same energy demanded at the head node. 


\subsection{Dynamics of the model}

Static models have no underlying temporal dimension. However, in the case of an integrated energy model, we have to account for the evolution of the system over time, as inventory is carried over from one time period to another.

Multiperiod network flow models may be viewed as a composition of multiple copies of a network, one at each point in time, with arcs that link these static snapshots describing temporal linkages in the system. With this construction, the size of the network is proportional to the number of periods.

If a unique time step is chosen to apply to the entire model, it must be small enough to capture the fastest dynamics of the integrated energy system, which are imposed by the electric energy subsystem. However, this results in unnecessary and counterproductive computations that take place for slower energy subsystems. Alternatively, one can take advantage of the fact that the integrated energy system is composed of different energy subsystems with distinct dynamics, define a different time step for each one, and thus eliminate the burden of redundant simulation details. As a result, different simulation time steps can be used for different energy subsystems.

Since the coal subsystem has relatively slow dynamics it is modelled using the largest time step (e.g., one week). Given the faster dynamics of the gas and petroleum subsystems, they are modelled using a smaller time step of, for example, one day. In the electric subsystem, the time steps are defined according to loading conditions, given by a segmented load duration curve used to represent the demand. Figure 4 illustrates this idea, where $T c, T e$, and $T g$ represent the last time step for the coal, the electric power, and the gas subsystems, respectively. In the gas subsystem, $1<x<y<T g$, and in the electricity subsystem $1<a<b<c<d<e<f<T e$. The arcs connecting the different time steps exist only in the fuel subsystems and represent the stock of fuel that is carried over from one period to the next. The oil subsystem is not illustrated in Figure 4 for simplicity but has a time step similar to the gas subsystem.

Figure 4 High-level representation of a multiperiod operation

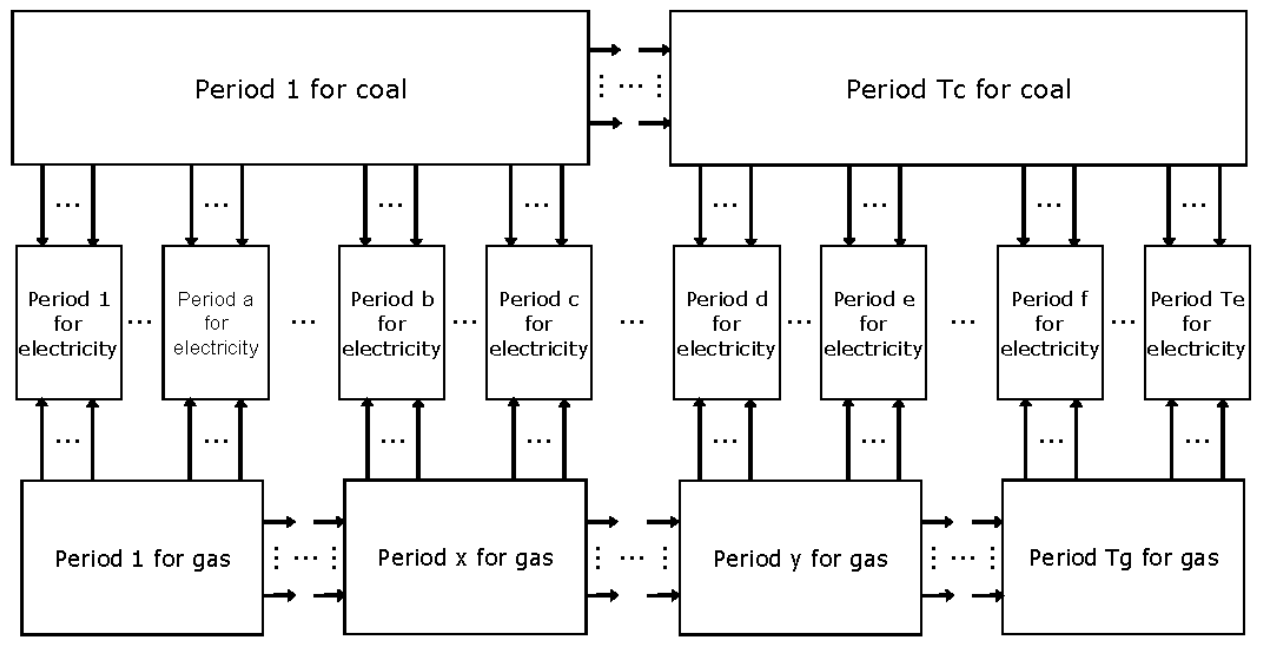




\subsection{Mathematical formulation}

Mathematically, the multiperiod generalised minimum cost flow problem is an optimisation model formulated as follows:

$$
\text { Minimise } z=\sum_{t \in T} \sum_{(i, j) \in M} \sum_{l \in L_{i j}} c_{i j}(l) e_{i j}(l, t)
$$

subject to:

$\sum_{\forall k} \sum_{l \in L_{j k}} e_{j k}(l, t)-\sum_{\forall i} \sum_{l \in L_{i j}} \eta_{i j}(l) e_{i j}(l, t)=b_{j}(t) \ldots \forall j \in N, \forall t \in T$,

$$
e_{i j \text { min }} \leq e_{i j}(t) \leq e_{i j \text { max }} \quad \forall(i, j) \in M, \forall t \in T .
$$

where $z$ is the objective function. The other symbols are described below.

\section{Parameters}

$b_{j}(t) \quad$ Supply (if positive) or negative of the demand (if negative) at node $j$, during time $t$

$c_{i j}(l) \quad$ Per unit cost of the energy flowing from node $i$ to node $j$, corresponding to the lth linearisation segment

$e_{i j \text { max }} \quad$ Upper bound on the energy flowing from node $i$ to node $j$

$e_{i j \text { min }} \quad$ Lower bound on the energy flowing from node $i$ to node $j$

$\eta_{i j}(l) \quad$ Efficiency parameter associated with the arc connecting node $i$ to node $j$, in the $l$ th linearisation segment

Variables

$e_{i j}(l, t) \quad$ Energy flowing from node $i$ to node $j$, corresponding to the $l$ th linearisation segment, during time $t$

Sets

$L_{i j} \quad$ Set of linearisation segments on the energy flowing from node $i$ to node $j$

$M \quad$ Set of arcs

$N \quad$ Set of nodes

$T \quad$ Set of indices of time periods

Indices

$i, j, k \quad$ Nodes.

The objective function in equation (1a) represents the total costs associated with the energy flows from the fossil fuel production sites to the electricity end users. These total costs are defined as the sum of the fuel production costs, the fuel transportation costs, the fuel storage costs, the electricity generation costs (operation and maintenance costs) and the electricity transmission costs.

The set of constraints in equation (1b) represent the conservation of flow constraints (energy balance constraints) for all nodes and for all times. For a particular node, the first term of this constraint is the total outflow of the node (flow emanating from the node) 
and the second term is the total inflow of the node (flow entering the node). The conservation of flow constraint states that the outflow minus the inflow must equal the supply/demand of the node. The set of constraints defined by equation (1c) are the flow bound constraints, which state that the flow must satisfy the lower bound and capacity of the respective arcs. The flow bounds represent the flows' operating ranges.

In matrix form, the problem can be represented as follows:

Minimise $z=\underline{c^{\prime}} \underline{e}$

subject to

$$
\begin{aligned}
& A \underline{e}=\underline{b} \\
& \underline{e}_{\min } \leq \underline{e} \leq \underline{e}_{\max } .
\end{aligned}
$$

In this formulation, $\boldsymbol{A}$ is an $n \times m$ matrix, called the node-arc incidence matrix of the generalised minimum cost flow problem. Each column of $\boldsymbol{A}$ is associated with a decision variable, and each row is associated with a node. The column $A_{i j}$ has a ' +1 ' in the $i$ th row, a ' -1 ' or a ' $-\eta_{i j}$ ' in the $j$ th row, and the rest of its entries are zero. For a detailed description on how the node-arc incidence matrix is formulated, including an illustrative example, please refer to Gil et al. (2003).

\section{Nodal prices}

The Karush-Kuhn-Tucker (KKT) conditions associated with the minimisation problem defined in the previous section yield the so called nodal prices (Fisher, 1981). They are usually explained as the shadow values related with each active constraint at the optimal solution of the choice variables and they represent the marginal costs of enforcing the constraints.

For simplicity, and without loss of generality, let us assume that the cost and efficiency parameters associated with each arc are constant functions. This permits the elimination of the parameter $l$, for notational simplicity. The Lagrangian function for equations (1a)-(1c) is then:

$$
\begin{aligned}
\mathrm{L}= & \sum_{t \in T} \sum_{(i, j) \in M} c_{i j}(t) e_{i j}(t)+\sum_{t \in T} \sum_{j \in N} \lambda_{j}(t)\left[\sum_{\forall k} e_{j k}(t)-\sum_{\forall i} \eta_{i j} e_{i j}(t)-b_{j}(t)\right]+ \\
& +\sum_{t \in T} \sum_{(i, j) \in M} \delta_{i j}(t)\left[e_{i j \cdot \min }-e_{i j}(t)\right]+\sum_{t \in T} \sum_{(i, j) \in M} \mu_{i j}(t)\left[e_{i j}(t)-e_{i j \cdot \max }\right]
\end{aligned}
$$

where $\lambda_{j}(t)$ is the Lagrangian multiplier associated with the energy balance constraint at node $j$ for time $t$. In other words, $\lambda_{j}(t)$ is the nodal price for node $j$, during time $t . \delta_{i j}(t)$ and $\mu_{i j}(t)$ are the Lagrangian multipliers associated with the lower and upper bound constraints, respectively, on the energy flowing from node $i$ to node $j$, during time $t$.

For optimality, in a given time period $t$, the relationship between the nodal prices of two linked nodes $i$ and $j$, is given by:

$$
\frac{\partial \mathrm{L}}{\partial e_{i j}}=c_{i j}+\lambda_{i}-\lambda_{j} \eta_{i j}-\delta_{i j}+\mu_{i j}=0 \text {. }
$$


From equation (4) we conclude that if the flow bound constraints are not binding $\left(\delta_{i j}=\mu_{i j}=0\right)$, the cost is zero $\left(c_{i j}=0\right)$, and there are no losses $\left(\eta_{i j}=1\right)$, then the nodal prices of the two linked nodes will be the same $\left(\lambda_{i}=\lambda_{j}\right)$.

\section{Numerical example}

\subsection{Single time step}

Suppose an energy system composed of two utilities, or two control areas, one in a northern region and the other in a southern region, interconnected by tie lines. The northern region operates two generating units: one oilfired and the other coalfired. The southern region operates three units: two coalfired and one natural gasfired. There are two possible suppliers of coal, one supplier of natural gas and one supplier of oil. Figure 5 shows the topology of the energy system in a network flow representation. The variables $x_{1}, \ldots, x_{8}$, represent the various fuel energy flows to the generating units. $x_{1}$ is measured in barrels, $x_{2}, \ldots, x_{7}$ are measured in tons, and $x_{8}$ is measured in thousand cubic feet (Mcf). On the balancing equations defined for each generator node, all of these units are converted to megawatthour (MWh), based on the heat value of each fossil fuel and the incremental heat rate of that particular generator. The variables $v_{1}, \ldots, v_{5}$ represent the electric energy output of the generating units and are expressed in MWh. Finally, the variables imp and exp represent the interchanged electric energy between the two regions, and are also expressed in MWh units. All of these variables $\left(x_{1}, \ldots, x_{8}, v_{1}, \ldots, v_{5}\right.$, imp, and exp) correspond to the energy flow variables $e_{i j}$ presented in the mathematical formulation described above. The data for the system are presented in Tables 1 and 2. For simplicity, assume that the fuel costs are fixed. Let's consider a single time step of one day (24 hours period) for the entire energy system.

Figure 5 Two-region system (single time step)

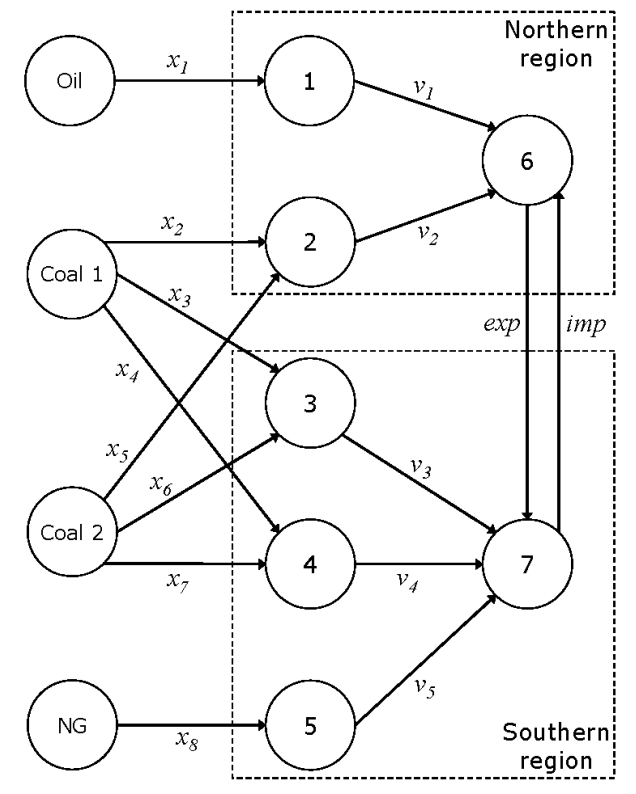


Nodal prices in an integrated energy system

Table 1 Unit characteristics and load

\begin{tabular}{lcccccc}
\hline Region & Unit & Fuel & Min $(\mathrm{MW})$ & Max $(\mathrm{MW})$ & $\begin{array}{c}\text { Incremental heat } \\
\text { rate }(\mathrm{MBtu} / \mathrm{MWh})\end{array}$ & $\begin{array}{c}\text { Average } \\
\text { load }(\mathrm{MW})\end{array}$ \\
\hline North & 1 & Oil & 150 & 600 & 9.95 & 1,200 \\
& 2 & Coal & 400 & 1,000 & 8.93 & \\
\multirow{5}{*}{ South } & 3 & Coal & 70 & 500 & 10.05 & 800 \\
& 4 & Coal & 70 & 500 & 10.05 & \\
& 5 & Gas & 0 & 300 & 9.55 & \\
\hline
\end{tabular}

Table 2 Fuel characteristics

\begin{tabular}{lcc}
\hline Supplier & Cost & Heat value \\
\hline Coal 1 & $30 \$ /$ ton & $11,500 \mathrm{Btu} / \mathrm{lb}$ \\
Coal 2 & $25 \$ /$ ton & $10,200 \mathrm{Btu} / \mathrm{lb}$ \\
Natural gas & $3.7 \$ / \mathrm{Mcf}$ & $1,000 \mathrm{Btu} / \mathrm{cf}$ \\
Oil & $21 \$ / \mathrm{barrel}$ & $1,43,500 \mathrm{Btu} / \mathrm{gallon}$ \\
\hline
\end{tabular}

The objective is to satisfy the demand at the minimum total operating costs, subjected to the conservation of energy constraints at all nodes and the units' operating ranges.

Several test cases were constructed to illustrate the impact that different situations have on the nodal prices of the integrated energy system, compared with the base case:

- base case: no limitations on the fuel transportation links and no limitation on the electric power transferred between the two regions

- test case 1: increase the southern region load, from $800 \mathrm{MW}$ to $1,100 \mathrm{MW}$

- test case 2: establish the cost of $1 \$ / \mathrm{MWh}$ on the electric energy transferred in either direction

- test case 3: consider a loss factor of $10 \%$ in the tie lines

- test case 4: limit the transfer power to $30 \mathrm{MW}$ in either direction

- $\quad$ test case 5: limit the coal delivered to units 3 and 4 from coal supplier $2\left(x_{6}+x_{7}\right)$ to 2,400 tons.

All of the results were obtained using the network optimiser routine of CPLEX 8.1 Division (http://www.cplex.com) in a $2.8 \mathrm{GHz}$ Pentium 4 processor with $1 \mathrm{~GB}$ of RAM. The computing time is negligible for all cases analysed. Table 3 presents the optimal energy flows and Table 4 displays the nodal prices obtained for each case. 
Table 3 Results of the optimisation problem

\begin{tabular}{lcccccc}
\hline & \multicolumn{7}{c}{ Optimal solution } \\
\cline { 2 - 7 } Energy flow & Base case & Test case 1 & Test case 2 & Test case 3 & Test case 4 & Test case 5 \\
\hline$x_{1}$ (barrel) & 5,944 & 11,887 & 5,944 & 5,944 & 6,736 & 5,944 \\
$x_{2}$ (ton) & 0 & 0 & 0 & 0 & 0 & 0 \\
$x_{3}$ (ton) & 0 & 0 & 0 & 0 & 0 & 3,393 \\
$x_{4}$ (ton) & 0 & 0 & 0 & 0 & 0 & 3,393 \\
$x_{5}$ (ton) & 10,506 & 10,506 & 10,506 & 10,506 & 10,506 & 10,506 \\
$x_{6}$ (ton) & 5,025 & 5,912 & 5,025 & 5,058 & 4,907 & 1,200 \\
$x_{7}$ (ton) & 5,025 & 5,912 & 5,025 & 5,058 & 4,907 & 1,200 \\
$x_{8}(\mathrm{Mcf})$ & 0 & 0 & 0 & 0 & 0 & 0 \\
$v_{1}(\mathrm{MWh})$ & 3,600 & 7,200 & 3,600 & 3,600 & 4,080 & 3,600 \\
$v_{2}(\mathrm{MWh})$ & 24,000 & 24,000 & 24,000 & 24,000 & 24,000 & 24,000 \\
$v_{3}(\mathrm{MWh})$ & 10,200 & 12,000 & 10,200 & 10,267 & 9,960 & 10,200 \\
$v_{4}(\mathrm{MWh})$ & 10,200 & 12,000 & 10,200 & 10,267 & 9,960 & 10,200 \\
$v_{5}(\mathrm{MWh})$ & 0 & 0 & 0 & 0 & 0 & 0 \\
imp $(\mathrm{MWh})$ & 1,200 & 0 & 1,200 & 1,333 & 720 & 1,200 \\
$\exp (\mathrm{MWh})$ & 0 & 2,400 & 0 & 0 & 0 & 0 \\
Total cost & 638.7 & 807.9 & 639.9 & 640.4 & 649.4 & 651.1 \\
(thousand $\$)$ & & & & & &
\end{tabular}

Table 4 Nodal prices for all cases

\begin{tabular}{lcccccc}
\hline & \multicolumn{7}{c}{ Nodal price $($ \$MWh) } \\
\cline { 2 - 7 } Node & Base case & Test case 1 & Test case 2 & Test case 3 & Test case 4 & Test case 5 \\
\hline 1 & 34.7 & 34.7 & 34.7 & 34.7 & 34.7 & 34.7 \\
2 & 10.9 & 10.9 & 10.9 & 10.9 & 10.9 & 10.9 \\
3 & 12.3 & 12.3 & 12.3 & 12.3 & 12.3 & 13.1 \\
4 & 12.3 & 12.3 & 12.3 & 12.3 & 12.3 & 13.1 \\
5 & 35.3 & 35.3 & 35.3 & 35.3 & 35.3 & 35.3 \\
6 & 12.3 & 34.7 & 13.3 & 13.7 & 34.7 & 13.1 \\
7 & 12.3 & 34.7 & 12.3 & 12.3 & 12.3 & 13.1 \\
\hline
\end{tabular}

For these test cases, the following observations are made:

- Base case. In the base case there are no limitations on the electric power transferred between the two regions, transmission costs are zero, and losses are neglected. As a result, the nodal price in the northern control area is equal to the nodal price in the southern control area. Furthermore, these nodal prices are equal to the nodal prices at nodes 3 and 4, associated with the coalfired units in the southern region, which are the marginal units for this case.

- $\quad$ Test case 1. Like in the base case, in this test case, losses are neglected, there are no congestions and the transmission costs are zero. Therefore, the nodal prices in both control areas are equal to one another. They are however higher than the nodal prices registered in the base case, as a result of the schedule of a generator with a higher incremental cost to supply the higher demand. The nodal prices at the control areas are now 34.7 \$/MWh, which is the incremental cost of the marginal unit (oilfired unit) for this case. 
- Test case 2. One of the requirements for equal nodal prices among interconnected control areas (zero transmission cost) is not satisfied in this test case. Since the northern control area is importing power, its nodal price is higher than the nodal price of the southern region, by the amount of the transmission incremental cost. That is, the nodal price of the southern control area is equal to the incremental cost of the marginal units $(12.3 \$ / \mathrm{MWh})$, while the nodal price of the northern region is equal to the incremental cost of the marginal units plus $1 \$ / \mathrm{MWh}$.

- $\quad$ Test case 3. This case tests a different violation of the requirements for equal nodal prices among interconnected control areas. Here, losses are different than zero. Since the northern control area is importing power, its nodal price is higher than the nodal price of the southern region. The nodal price of the southern control area is equal to the incremental cost of the marginal units $(12.3 \$ / \mathrm{MWh})$, while the nodal price of the northern region is equal to the incremental cost of the marginal units weighted by the inverse of the efficiency rate $(12.3 / 0.9=13.7 \$ / \mathrm{MWh})$.

- Test case 4. In this case, congestion is artificially created in the electric subsystem by limiting the capacity of the tie line to $30 \mathrm{MW}$. This again violates one of the requirements for equal nodal prices among interconnected control areas, making the nodal price of the northern control area equal to the incremental cost of the marginal unit in the north (34.7 \$/MWh corresponding to the oilfired unit) and the nodal price of the southern control area equal to the incremental cost of the marginal units in the south (12.3\$/MWh corresponding to the coalfired units).

- $\quad$ Test case 5 . In test case 5, congestion is artificially created in the coal delivered to units 3 and 4 from coal supplier 2. The nodal prices in the two control areas are still equal to one another; since there is no congestion in the tie line, losses are neglected, and the transmission cost is zero. Furthermore, they are equal to the nodal prices at the marginal units (still units 3 and 4). However, they are now higher than the base case due to the congestion in the coal delivery system, which results in the utilisation of coal delivery through an alternative route with associated higher cost. The next increment of electric energy in either control area is supplied by units 3 and 4, through coal delivered from coal supplier 2.

From these results we observe that, in the absence of congestion, losses and costs in the transmission lines (base case, test case 1, and test case 5), the nodal prices are the same among interconnected control areas. When demand increases (test case 1), the nodal prices at the control areas may increase as a result of the schedule of units with higher incremental costs. Furthermore, when a constraint in the fuel production or transportation systems becomes binding (test case 5), its marginal enforcement cost, and hence its impact on the nodal prices at affected control areas, can be significant.

Test cases 2-4 illustrate situations that may result in different nodal prices in interconnected control areas.

In all cases, the nodal price in a particular control area of an integrated energy system may be interpreted as the marginal cost of supplying the next increment of energy at that specific location, considering not only the physical aspects of the electric generation and transmission system, but also the physical aspects of the various fuel production and delivery systems. More generally, the nodal prices represent the opportunity cost of energy at each node of the integrated energy system and can therefore be used to induce 
an efficient use of all the resources involved, to identify the crucial interdependencies between the electric energy subsystem and the various fuel production and delivery subsystems, and to provide correct economic signals for infrastructure investments.

\subsection{Multiple time steps}

To exemplify the ability of the model to deal with multiple time steps and longer time periods, we now consider the energy system presented above for a one-year time period, with the electric subsystem disaggregated into hourly steps, the oil and the gas subsystems disaggregated into daily steps, and the coal subsystem disaggregated into weekly steps. Two storage facilities are included, one for oil and the other for natural gas, which establish a dependency on the flows among different time steps. The oil and the natural gas storage tanks have capacities of 1,00,000 barrels and one million cubic feet, respectively. The loads follow the demand curves presented in Figure 6 and the prices for natural gas and oil are shown in Figures 7(a) and 7(b), respectively. The prices for coal are those presented in Table 2 above and remain constant throughout the year. The tie line capacity is limited to $100 \mathrm{MW}$ in either direction and for the entire simulation period.

Figure 6 Hourly load curves

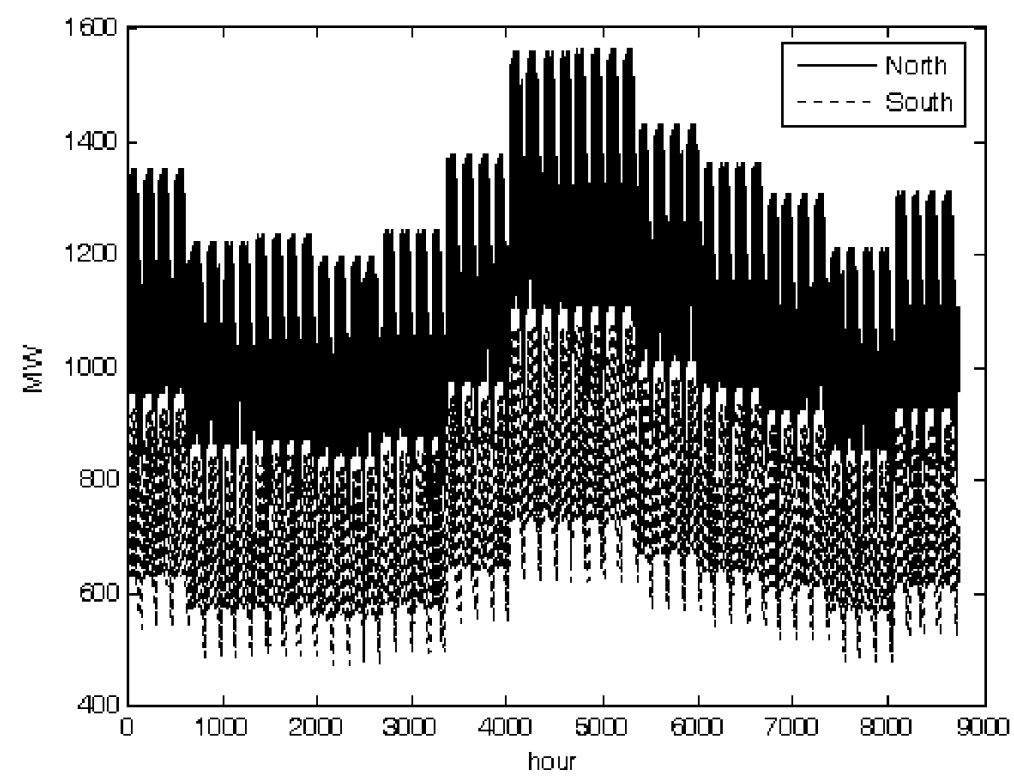

Using the same computation resources as described above, an optimal solution has been reached within 2.87 seconds. Figures 8 and 9 present some of the optimal energy flows in the electric subsystem. Figure 8 shows the schedules for the generating units and Figure 9 shows the tie line flows, where positive values represent imports (electric energy flowing from node 7 to node 6). Although the model generates hourly values for the electric subsystem, we aggregated them weekly for clarity. 
Figure 7 Daily natural gas and oil prices: (a) natural gas and (b) oil

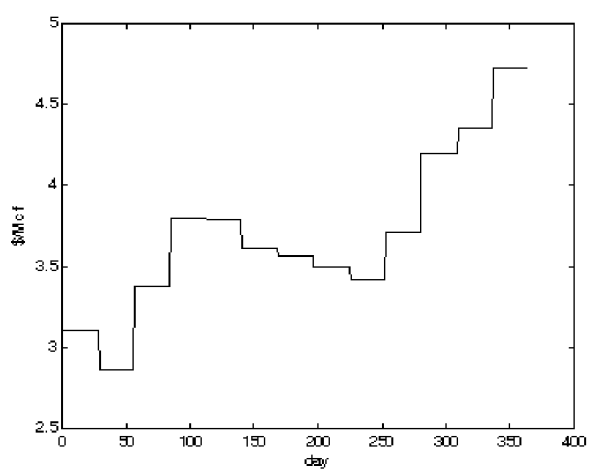

(a)

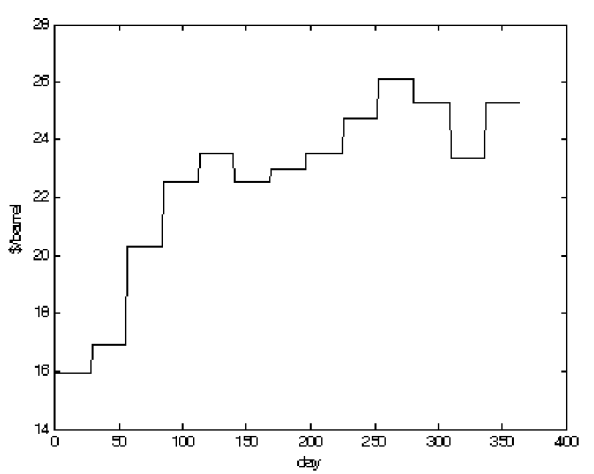

(b)

Figure 8 Weekly average scheduling of the generating units

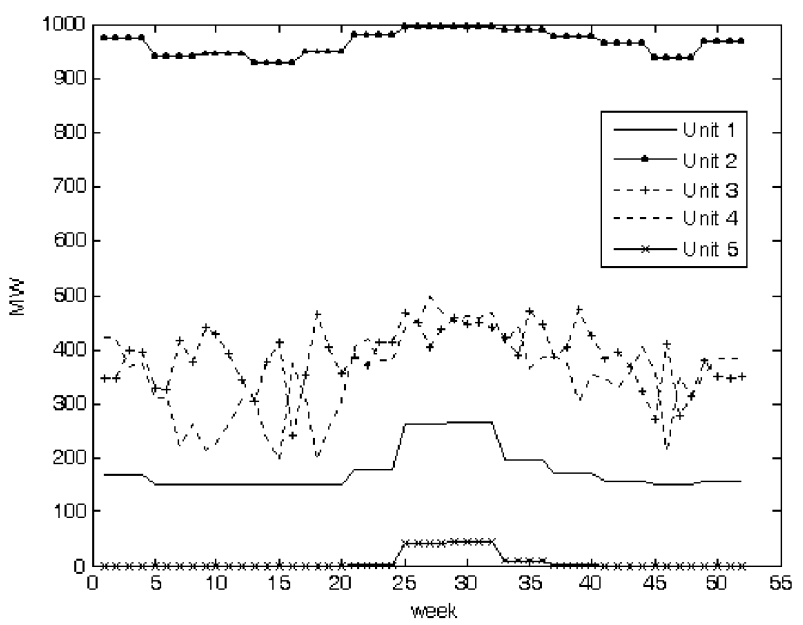

Figure 9 Weekly average tie line schedule (positive values represent imports)

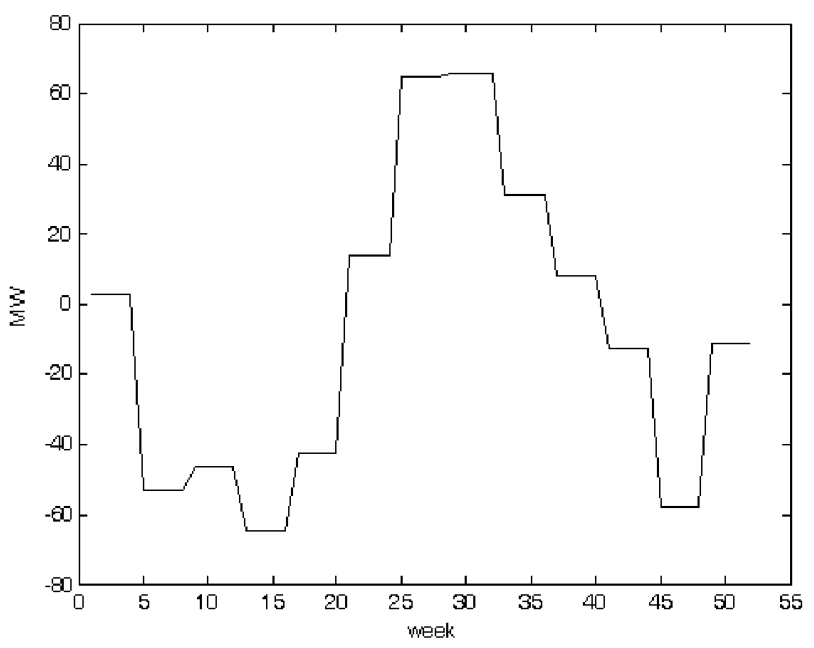


The temporal variation of the nodal prices in the fuel subsystems (nodes 1-5) and in the control areas (nodes 6 and 7) are presented in Figures 10 and 11, respectively.

Figure 10 Weekly average nodal prices in the generating units

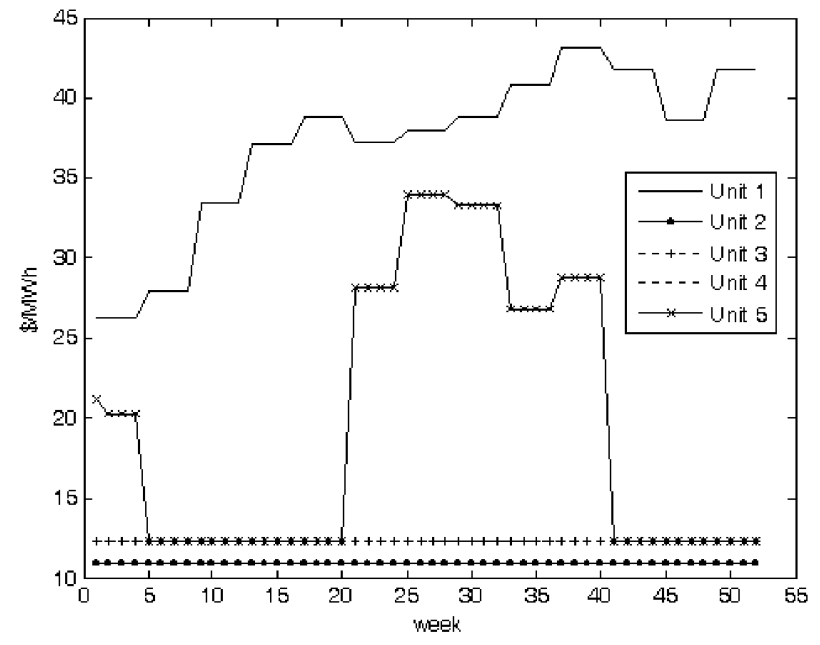

Figure 11 Weekly average nodal prices in the control areas

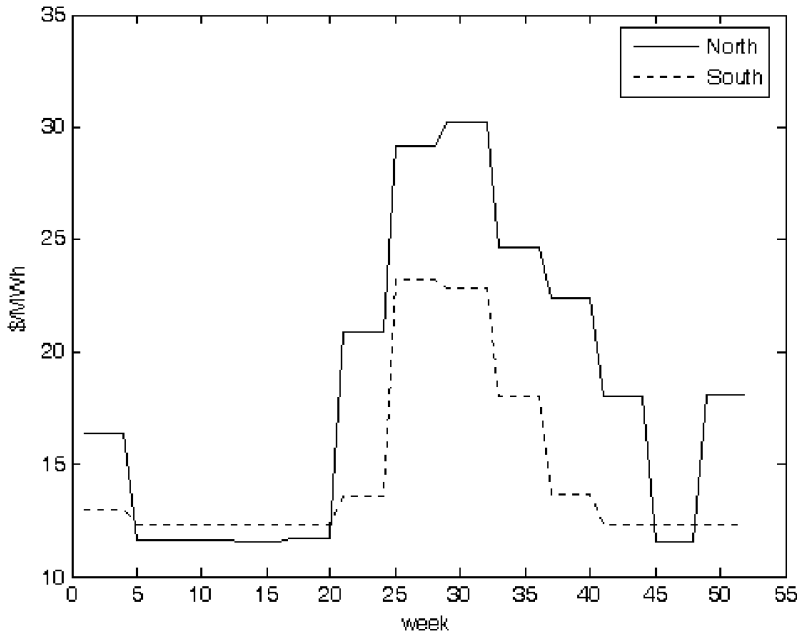

Since there are no variations in the coal prices, the nodal prices for units 2,3 , and 4 remain constant for the entire period. As expected, the nodal price at unit 2 is 10.9\$/MWh and the nodal prices at units 3 and 4 are $12.3 \$ / \mathrm{MWh}$. Since there are natural gas and oil price changes, the nodal prices at units 1 and 5 vary throughout the year. The nodal prices for the oil unit (unit 1) follow closely the variations of the oil prices presented in Figure 7, but the same does not happen for the natural gas unit (unit 5). This is because natural gas is being stored in the reserve tank when gas prices are low and used when this fuel price is high. Although the same happens with the oil storage facility, its capacity is not big enough to allow us to observe these dynamics in flattening the nodal prices for unit 1 . 
For some hours during the year the nodal prices are the same in both control areas, but this is not visible in Figure 11, because only the weekly averages are plotted. Nonetheless, it is important that they are equal to one another in all time periods for which the tie line capacity is not binding the solution. The higher nodal prices that occur during weeks $1-5,20-45$, and 50-52 are associated with the periods in which loads are higher (see Figure 6), which result in the commitment of units with higher incremental costs. Despite the load increase observed in the last weeks of the year for both control areas, the nodal price in the Southern region did not rise due to the activity that took place in the natural gas storage facility.

\section{Conclusion}

Although economic and physical performances of individual subsystems are well studied and understood, there has been little effort to study its global characteristics. Consequently, each energy subsystem supports specific procedures and strategies according to their own value system (i.e., economic, technical, political, and environmental constraints), which may be fragmentary because they are missing the necessary consolidation in global actions or alternative strategies for an efficient operation.

This paper extends the concept of nodal prices to the integrated energy system, i.e., an energy system that comprises not only the generation and transmission network, but also the fuel production and delivery networks. The nodal price information provides an indication of the economic interdependencies between the fuel networks and the electric network, which is key information for an economically efficient operation of the energy system. They support decision makers (in particular, policy makers) in creating the necessary conditions so that investments in the fuel networks are made in an efficient way, i.e., in a way that most affects the prices in the electric network.

The model proposed in this paper to optimise the medium term operation of an integrated energy system is a multiperiod generalised network flow model. The benefits of using a network flow modelling technique rather than a more general linear programming approach are associated with the fact that more efficient solution procedures can be used, which is of importance due to the high dimension that characterises an integrated energy system.

Another unique characteristic of this modelling approach is that it does not require the same time step to be used in all energy subsystems. As shown in the example presented in Section 4.2, where the electric subsystem was disaggregated into hourly steps and the various fossil fuel subsystems into daily and weekly steps, each energy subsystem can be represented according to its dynamics.

\section{Acknowledgement}

This work was partially supported by the Fundação para a Ciência e a Tecnologia (FCT), Portugal, under Grant SFRH/BD/8079/2002. 


\section{References}

Ahuja, R.K., Magnanti, T.L. and Orlin, J.B. (1993) Network Flows, Prentice-Hall, Inc., Englewood Cliffs, NJ.

Arroyo, J.M. and Conejo, A.J. (2000) 'Optimal response of a thermal unit to an electricity spot market', IEEE Transactions on Power Systems, Vol. 15, No. 3, August, pp.1098-1104.

Bazaraa, M.S., Jarvis, J.J. and Sherali, H.D. (1990) Linear Programming and Network Flows, John Wiley \& Sons, Inc., New York.

Chen, L., Suziki, H., Wachi, T. and Shimura, Y. (2002) 'Components of nodal prices for electric power systems', IEEE Transactions on Power Systems, Vol. 17, No. 1, February, pp.41-49.

Ecker, J.G. and Kupferschmid, M. (1991) Introduction to Operations Research, Krieger Publishing Company, Malabar, FL.

Energy Information Administration (1998) 'Challenges of electric power industry restructuring for fuel suppliers', DOE/EIA-0623(98), Washington DC, September.

Energy Information Administration (2000) 'The changing structure of the electric power industry 2000: an update', DOE/EIA-0562(00), Washington DC, October.

Energy Information Administration (2004) 'Annual energy review 2003', DOE/EIA-0384(2003), Washington DC, September.

Fisher, M.L. (1981) 'The Lagrangian relaxation method for solving integer programming problems', Management Science, Vol. 27, No. 1, pp.1-18.

Gil, E.M., Quelhas, A.M., McCalley, J.D. and Voorhis, T.V. (2003) 'Modeling integrated energy transportation networks for analysis of economic efficiency and network interdependencies', Proceedings of the 2003 NAPS Conference, October, University of Missouri, Rolla, MO.

Glover, F., Klingman, D. and Phillips, N.V. (1992) Network Models in Optimization and Their Applications in Practice, John Wiley \& Sons, Inc., New York.

Hong, Y.Y. and Weng, M.T. (1999) 'Investigation of nodal prices in a deregulated competitive market - case studies', Proceedings of the 1999 PowerTech Budapest 99, International Conference, p.161.

Jensen, P.A. and Bard, J.F. (2002) Operations Research Models and Methods, Wiley Text Books, September, New York, NY.

Knight, J.C., Elder, M.C., Flinn, J. and Marx, P. (1998) Analysis of Four Critical Infrastructure Applications, Computer Science Report No. CS-97-27, University of Virginia, September.

Litvinov, E., Zheng, T., Rosenwald, G. and Shamsollahi, P. (2004) 'Marginal loss modeling in LMP calculation', IEEE Transactions on Power Systems, Vol. 19, No. 2, May, pp.880-888.

Moslehi, K., Sherkat, V.R. and Cacho Ruiz, F. (1991) 'Optimal scheduling of long-term fuel purchase, distribution, storage and consumption', Proceedings of the Power Industry Computer Application Conference, Baltimore, MD, pp.98-104.

Motto, L., Galiana, F.D., Conejo, A.J. and Arroyo, J.M. (2002) 'Network-constrained multiperiod auction for a pool-based electricity market', IEEE Transactions of Power Systems, Vol. 17, No. 3, August, pp.646-653.

Ott, L. (2003) 'Experience with PJM market operation, system design, and implementation', IEEE Transactions on Power Systems, Vol. 18, No. 2, May, pp.528-534.

Rinaldi, S.M., Peerenboom, J.P. and Kelly, T.K. (2001) 'Identifying, understanding, and analyzing critical infrastructure interdependencies', IEEE Control Systems Magazine, Vol. 21, December, pp.11-25.

Shahidehpour, M., Yamin, H. and Li, Z. (2002) Market Operations in Electric Power Systems, John Wiley \& Sons, Inc., New York, Chapter 10.

Sterman, J.D. (2000) Business Dynamics: Systems Thinking and Modeling for a Complex World, Irwin, McGraw-Hill, Chapter 3, New York, NY.

Sun, D. (2002) 'LMP and FTR in the standard market design', Proceedings of the 2002 IEEE Power Engineering Society Summer Meeting, Vol. 3, pp.1269-1270. 
Tabors, R.D. (2001) 'Forward markets for transmission that clear at LMP: a hybrid proposal', Proceedings of the 2001 System Sciences 34th Annual Hawaii International Conference, Maui, HI, p.8.

Vermuru, S. and Lemonidis, L. (1990) Fuel Scheduling, a paper in the IEEE Power Engineering Society tutorial special publication 90EH0238-5-PWR, 'Application of optimization methods for economy/security functions in power system operations', IEEE Power Engineering Society.

Wood, J. and Wollenberg, B.F. (1996) Power Generation, Operation and Control, 2nd ed., John Wiley \& Sons, Inc, New York.

\section{Websites}

CPLEX Division (1998) CPLEX Optimization Package, ILOG Inc., USA. [Online] Available: http://www.cplex.com.

North American Electric Reliability Council, Resources Subcommittee. [Online] Available: http://www.nerc.com/ filez/rs.html. 